\title{
Digital computation and in situ STM approach of silicon anisotropic etching
}

\author{
Jérôme Kasparian ${ }^{\mathrm{a}, \mathrm{b}}$, M. Elwenspoek ${ }^{\mathrm{c}}$, Philippe Allongue ${ }^{\mathrm{a}, *}$ \\ ${ }^{a}$ Laboratoire de Physique des Liquides et Électrochimie, UPR 15 CNRS associé à l'ESPCI, 10 Rue Vauquelin, F-75005 Paris, France \\ ${ }^{\circ}$ Laboratoire de Spectrométrie Ionique et Moléculaire, UMR 5579 CNRS, Université Claude-Bernard, Bât. 205, \\ 43 Bd du II Novembre 1918, F-69622 Villeurbanne, Cedex, France \\ ' MESA Research nstitute, University of Twente, PO Box 217, NL-7500 AE Enschede, Netherlands
}

Received 7 February 1997; accepted for publication 28 April 1997

\begin{abstract}
Si anisotropic etching is simulated on the atomic level with a simple algorithm (Monte Carlo method). The comparison of simulated sequences with in situ real-time STM observations of $\mathrm{n}-\mathrm{Si}(111)$ in $\mathrm{NaOH}$ demonstrates the relevance of the model. Analytical expressions for the growth of triangular etch pits are given and a method proposed to determine experimentally the reaction rates on the atomic scale. The bias dependence of reaction rates and the mechanism of nucleation of etch pits are also discussed in the framework of the chemical description of Si etching. 1997 Elsevier Science B.V.
\end{abstract}

Keywords: Computer simulations; Etching; Electrochemical methods; Scanning tunneling microscopy; Semiconductor-electrolyte interfaces; Silicon; Single crystal surfaces; Solid liquid interfaces

\section{Introduction}

Silicon etching is an important step in the microelectronics industry and recent work has addressed the question of the relationship between the etching parameters (solution composition, doping level and type, etc.) and surface microstructures (see Ref. [1] for a review, and Refs. [2-5]). For applications, an atomistic model capable of predicting the microstructures of chemically etched silicon surfaces would be highly desirable, but results remain rather empirical. A full theoretical description is certainly difficult to achieve because the removal of a single atom from the crystal surface is a

\footnotetext{
* Corresponding author. Fax: (+33) 143361680 ; e-mail: pa@ccr.jussieu.fr
}

complex event when referring to the reaction mechanism of Fig. 1 [4]. Among the theoretical work, ab initio calculations on clusters have yielded insights into the kinetic origin of the $\mathrm{H}$ termination of Si surfaces [6]. Recent Monte Carlo computation, focusing on the probability of breaking $\mathrm{Si}-\mathrm{Si}$

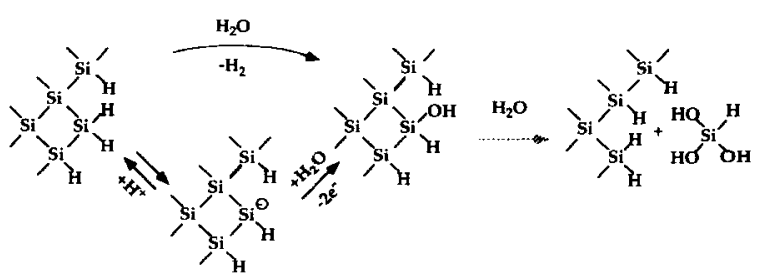

Fig. 1. Reaction model describing the initial steps of Si etching (after Ref. [4]). The top route is purely chemical, the bottom one is electrochemical (free carriers exchanged). 
bonds, accounted successfully for many aspects of the orientational dependence of Si etching in alkaline solutions [7].

In alkaline media, where micromachining has been the main purpose, experimental data are mainly macroscopic and concern the unusual orientational etching rate $[8,9]$. The influence of the solution composition (water activity, rôle of the cations and of organic additives) deserves more atomic-scale characterization. One STM work studied the effect of applied bias and the rôle of an organic additive in $\mathrm{NaOH}$ on the atomic scale [10]. By contrast, the possibility of preparing atomically flat (111) surfaces in $\mathrm{NH}_{4} \mathrm{~F}$ solutions has prompted a great deal of work and many atomic-scale correlations are available. The tilt of the (111) surface is known to be critical to prepare ordered staircase structures $[1,2,11-13]$. The optimal value of the miscut angle depends on the solution composition and applied potential in order to find a compromise between the nucleation rate of etch pits and the speed with which the atomic steps migrate laterally. The $\mathrm{pH}$ value [2], the presence of additives [10] and the doping level [5] are also important parameters. With $\mathrm{Si}(100)$ the problem of preparing flat surfaces is not completely solved yet, despite excellent results obtained in $\mathrm{HF} / \mathrm{HCl}$ [14].

The present paper combines Monte Carlo simulations and in situ STM observations to determine quantitatively the rate of $\mathrm{Si}$ dissolution on the atomic scale. With respect to our preliminary study [15], this work is a more thorough study in which specific experiments are presented to derive independently the different reaction rates on the atomic scale. We also provide a direct validation of the algorithm used by comparing the experimental variations of triangular etch pits with analytical expressions of their growth. Implications and consistency of results with Fig. 1 are also discussed.

\section{Simulation of Si dissolution}

\subsection{Atomistic model}

The atomistic model assumes that the removal of an $\mathrm{Si}$ atom is uniquely related to its surface coordination. According to Fig. 1, the disruption of an $\mathrm{Si}-\mathrm{Si}$ back-bond binds new $\mathrm{H}$ atoms on the surface and the $\mathrm{Si}$ atom progressively goes into solution as a complex $\operatorname{SiH}_{n}(\mathrm{OH})_{m}$ (with $n+m=$ 4 ). The departing $\mathrm{Si}$ atom leaves the crystal irreversibly (no redeposition occurs) and surface diffusion is not taken into consideration. In the following, a surface site is an atom identified by the number of $\mathrm{Si}-\mathrm{H}$ bonds and their orientation with respect to the surface plane. A monolayer $\mathrm{Si}(111)$ crystal is considered here, which proved to be sufficient to investigate the very initial stages of $\mathrm{Si}$ anisotropic etching. A three-dimensional model is currently being developed to deal with etching on any crystallographic face [16].

Fig. 2 shows the atomic structure of a $(1 \times 1)$ H-terminated $\mathrm{Si}(111)$ surface from the top [4]. This surface chemistry has been observed in $\mathrm{NH}_{4} \mathrm{~F}$ or $\mathrm{NaOH}[17,18]$. Filled circles are $\mathrm{H}$ atoms and open circles are Si topmost atoms. The terrace atoms are threefold coordinated with the lattice and have a single $\mathrm{Si}-\mathrm{H}$ bond normal to the surface. Three different sites exist at step edges. Step edge atoms may also be triply bound to the crystal, but with the $\mathrm{Si}-\mathrm{H}$ bond nearly in-plane. Kink sites have two bonds with the lattice and two $\mathrm{Si}-\mathrm{H}$ bonds, the latter two being either in the vertical

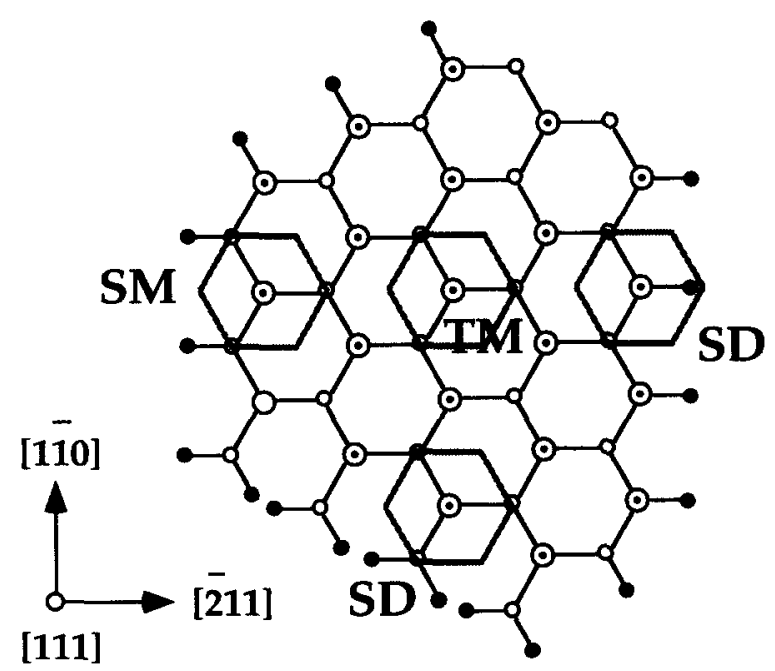

Fig. 2. Atomic model (top view) of the H-terminated Si(111) surface. The hexagonal sites are those considered in computation. They have the reactivity of the least stable atom: terrace monohydrides (TM), step monohydrides (SM) and step dihydrides (SD). 
plane or almost in-plane. Following Chabal's assignment, terrace atoms are referred to as TM sites for terrace monohydrides, and step edge sites are referred to as SM and SD for step monohydrides and step dihydrides respectively. No distinction is made between the two possible SD configurations because this proved to be unnecessary. Note that SM and SD atoms have the coordination of atoms on ideal (110) and (100) surfaces respectively.

In computation, TM, SM and SD sites are hexagons centered on terrace Si topmost atoms. Each includes, on the average, the related abovedefined sites as the least stable atom (Fig. 2). The $\mathrm{SM}$ site consists, for instance, of one terrace $\mathrm{Si}$ monohydride and $2 \times \frac{1}{2}$ step Si monohydrides, i.e. one on the average. The SD site is made of one step Si dihydride and one vertical monohydride. TM sites have only one terrace Si monohydride. Tests with algorithms involving atoms instead of hexagonal sites have shown no difference. We therefore make no distinction between computational and atomic sites in the following.

The algorithm of simulation is described in Appendix A. Reaction rate $k_{i}$ values on sites of type $i=\mathrm{TM}, \mathrm{SM}, \mathrm{SD}$ are expressed in reciprocal seconds. The time given in the simulation is therefore expressed in seconds. The simulation cell is a parallelogram to grow the largest possible triangular etch pit. It has $150 \times 150$ sites which corresponds to an $\mathrm{Si}(111)$ surface of edge $576 \AA$ (i.e. about $\frac{1}{4}$ of experimental images).

\subsection{Critical length of a kink free step}

On a perfectly straight infinite step (SM-terminated), the removal of an SM atom generates two kink sites (SD sites) which propagate in opposite directions along the edge (Fig. 3). The critical length is defined as the statistical distance separating the two SD kinks when a second defect is created on the newly exposed edge.

Under the assumptions of the model, at time $t$ the length of the newly exposed edge is $L=2 \delta k_{\mathrm{SD}} t$ if the $\mathrm{SM}$ atom has been removed at $t=0 . \delta=3.84 \AA$ is the distance between two SM sites on the step edge. The mean nucleation rate of a second defect on the newly exposed step edge is therefore $N(t) k_{\mathrm{SM}}$, with $N(t)=L / \delta=2 k_{\mathrm{SD}} t$
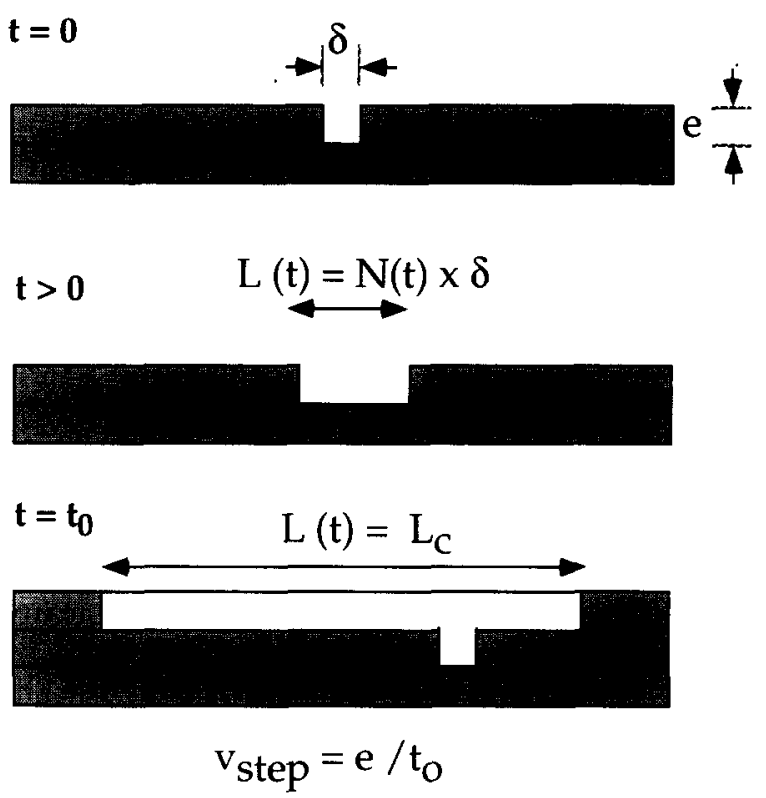

Fig. 3. Schematic model explaining the critical length $L_{\mathrm{c}}$.

the number of atoms on this step. On the average, therefore, the second defect appears on the same step after a time lag $\Delta t_{0}$ defined by the equation $2 \int_{0}^{\Delta t_{0}} k_{\mathrm{SD}} k_{\mathrm{SM}} t \mathrm{~d} t=1$. It follows that $\Delta t_{0}=1 / \sqrt{ } k_{\mathrm{SD}} V k_{\mathrm{SM}}$ and that the critical length is $L_{\mathrm{c}}=2 \delta k_{\mathrm{SD}} \Delta t_{0}=2 \delta /\left(\mathrm{V} k_{\mathrm{SD}} \sqrt{ } k_{\mathrm{SM}}\right)$.

\subsection{Growth modes of triangular etch pits}

Let us consider a triangular etch pit of edge length $L$. If $L<L_{\mathrm{c}}$, the two kink sites propagate and reach the corner of the triangle before another SM atom has been removed on the same edge. The frequency of removal of one SM atom on this edge is $F(t)=k_{\mathrm{SM}} L(t) / \delta$ (there are $N(t)=L(t) / \delta$ atoms on a step edge of length $L(t))$. Writing that $\mathrm{d} L(t) / \mathrm{d} t=3 \delta F(t)$ (the factor 3 comes from the fact that etch pits are equilateral triangles), it follows that $L(t)$ is the solution of the equation $\mathrm{d} L(t) / \mathrm{d} t=3 L(t) k_{\mathrm{SM}}$ and reads

$L(t)=\dot{\delta} \exp \left(3 k_{\mathrm{SM}} t\right)$.

When $L(t)>L_{\mathrm{c}}$, a second SM atom is removed on the same edge before the two kinks have reached the corner of the triangle (Fig. 4(b)). The step edge is no longer ideal (kink free) and retracts 
(a)

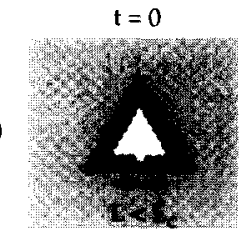

(b)

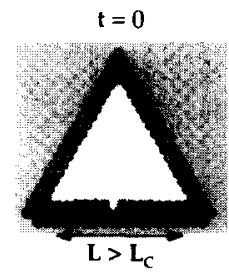

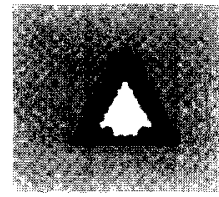

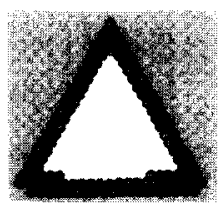

$t$

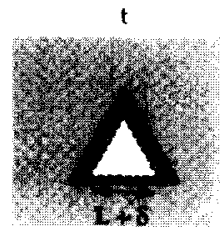

$\mathbf{t}=\mathbf{t}_{0}$

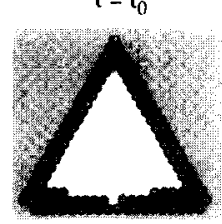

Fig. 4. Scheme showing the growth of a triangular etch pit under two extreme conditions. (a) When $L<L_{\mathrm{c}}, L \approx \exp \left(3 k_{\mathrm{SM}} t\right)$. (b) When $L>L_{\mathrm{c}}, L \approx V k_{\mathrm{SM}} \sqrt{ } k_{\mathrm{SD}} t+C$ (see text).

at an average speed $v_{\text {Step }}=e / \Delta t_{0}$, with $\Delta t_{0}$ defined above and $e$ the distance between two atomic rows running parallel to the step edge. In that case $L(t)$ is the solution of equation $\mathrm{d} L(t) / \mathrm{d} t=$ $3 \delta \sqrt{ } k_{\mathrm{SD}} \sqrt{ } k_{\mathrm{SM}}$ and reads

$$
L(t)=3 \delta \sqrt{ } k_{\mathrm{SD}} \sqrt{ } k_{\mathrm{SM}} t+C
$$

with $C$ a constant. In conclusion, the model predicts that triangular etch pits should grow exponentially in the initial stages before a linear regime is observed as the time increases. The following will show that Eqs. (1) and (2) are in fact well accounted for by experimental observations. They also suggest a method, based on the observation of the growth of a bilayer deep triangle on the (111) face, to directly measure the rate constants $k_{\mathrm{SD}}$ and $k_{\mathrm{SM}}$.

\section{Experimental}

In situ STM images were acquired with a homebuilt microscope [19]. Tungsten tips were etched electrochemically in $\mathrm{NaOH}$ and covered with apiezon wax, leaving the very end wax-free. The reference electrode was a quasi-Pd-hydrogen electrode, and the counter electrode was a Pt wire. Tunnel currents ranged between 0.2 and $1 \mathrm{nA}$. Images are presented as unfiltered gray scale top views, with, unless otherwise specified, heights decreasing from white to black. Si samples were cut from $0.5 \Omega \mathrm{cm}$

n-Si(111) wafers (Siltronix, France) tilted by $0.7^{\circ}$ in the [112] direction. As-received samples were cleaned in hot trichlorethylene, acetone and methanol before oxide stripping in $40 \% \mathrm{HF}$ (for $1 \mathrm{~min}$ ) and etching in $40 \% \mathrm{NH}_{4} \mathrm{~F}$ (for $3 \mathrm{~min}$ ). A rear ohmic contact was ensured with In-Ga alloy. Solutions were prepared with reagent-grade chemicals and bidistilled water.

\section{Results}

\subsection{Quantitative analysis of STM observations}

Fig. 5 is a time sequence showing the etching of $\mathrm{n}-\mathrm{Si}(111)$ at cathodic bias in $\mathrm{NaOH}$ ( $I=$ $\left.-200 \mu \mathrm{A} \mathrm{cm}^{-2}\right)$. The process is entirely anisotropic at this bias (no pitting observed) because the electrons accumulated at the surface suppress the electrochemical route in Fig. 1 [4]. All steps and etch pits are respectively one bilayer high and deep (3.1 ̊). Triangular etch pits have straight edges parallel to the [110] direction. (The growth of triangles A, B and C is analyzed below in Fig. 13.) Following the method given in Ref. [19], the etch rate is estimated to be $0.12 \AA \mathrm{min}^{-1}$ in the sequence.

That the very initial stages of formation of etch pits $\mathrm{B}$ and $\mathrm{C}$ are resolved is rather rare to observe (see ticks in the top-left image in Fig. 5). Following the growth of triangles over a long period of time requires also several favorable conditions, like a very stable tip and wide terraces to avoid etch pits merging too rapidly into each other or into steps. The speed of growth of triangles must also be compatible with the scan frequency of the microscope (in this study images are typically recorded in $50 \mathrm{~s}$ ). Repeating the experiment shown in Fig. 5 requires either re-preparing the surface from the beginning or preparing it in situ to start with perfect terraces.

Long-standing observations are impossible at the rest bias because the surface of the $\mathrm{n}-\mathrm{Si}$ electrode is depleted of electrons (for a review on STM on semiconductors see Ref. [20]). Fig. 6 describes a technique to overcome this difficulty and to investigate the details of the nucleation of etch pits. The following technique was used. The first image was recorded with the sample polarized 

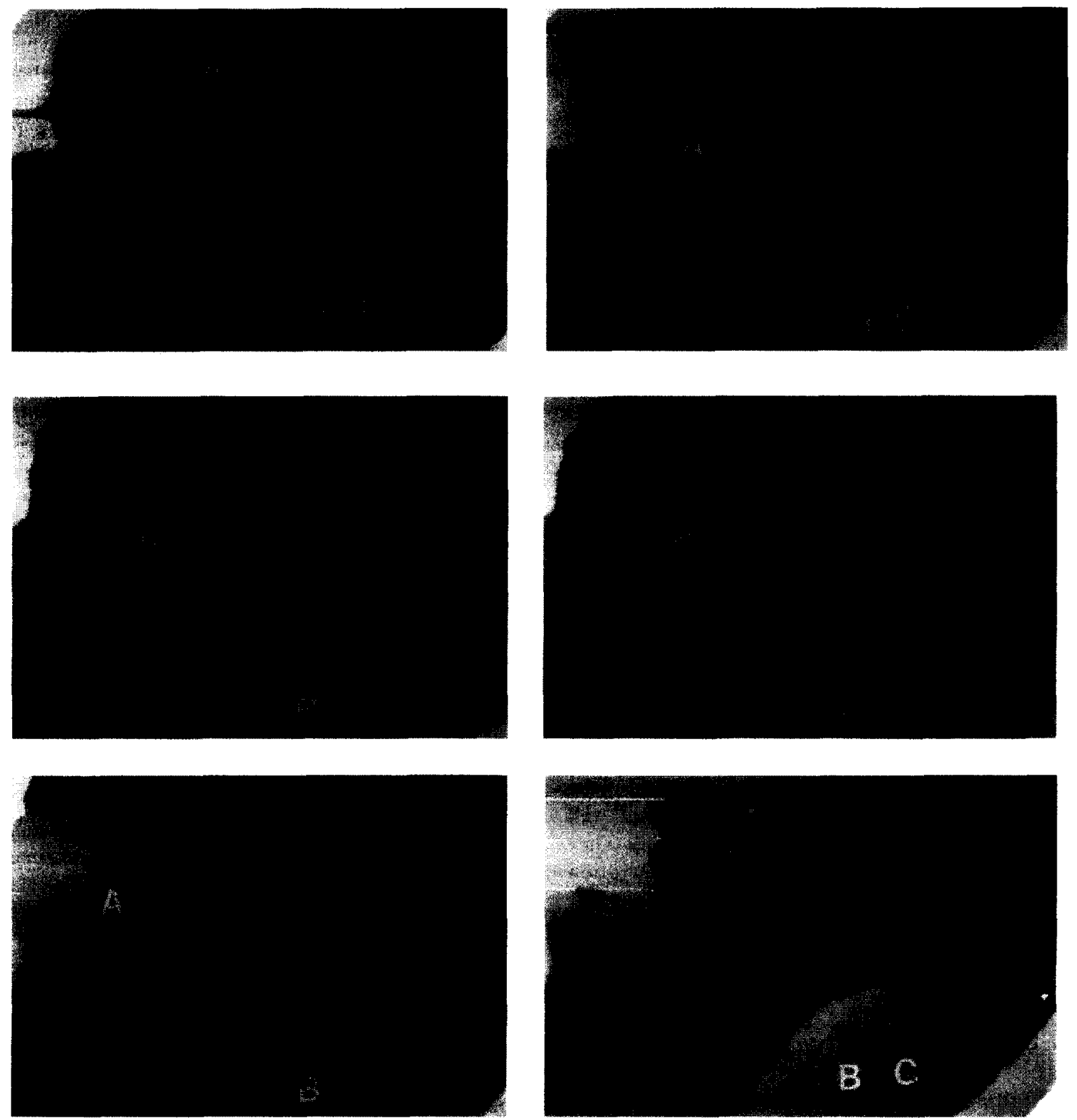

Fig. 5. In situ STM sequence recorded on $\mathrm{n}-\mathrm{Si}(111)$ at cathodic bias in $\mathrm{NaOH}$ (hydrogen current $200 \mu \mathrm{A} \mathrm{cm}{ }^{-2}$ ). The growth of the triangular pits A-C is analyzed in Fig. 13. Frames are $1200 \times 1000 \AA^{2}$. The ticks in the top-left image mark the nucleation of triangles $B$ and $C$. 

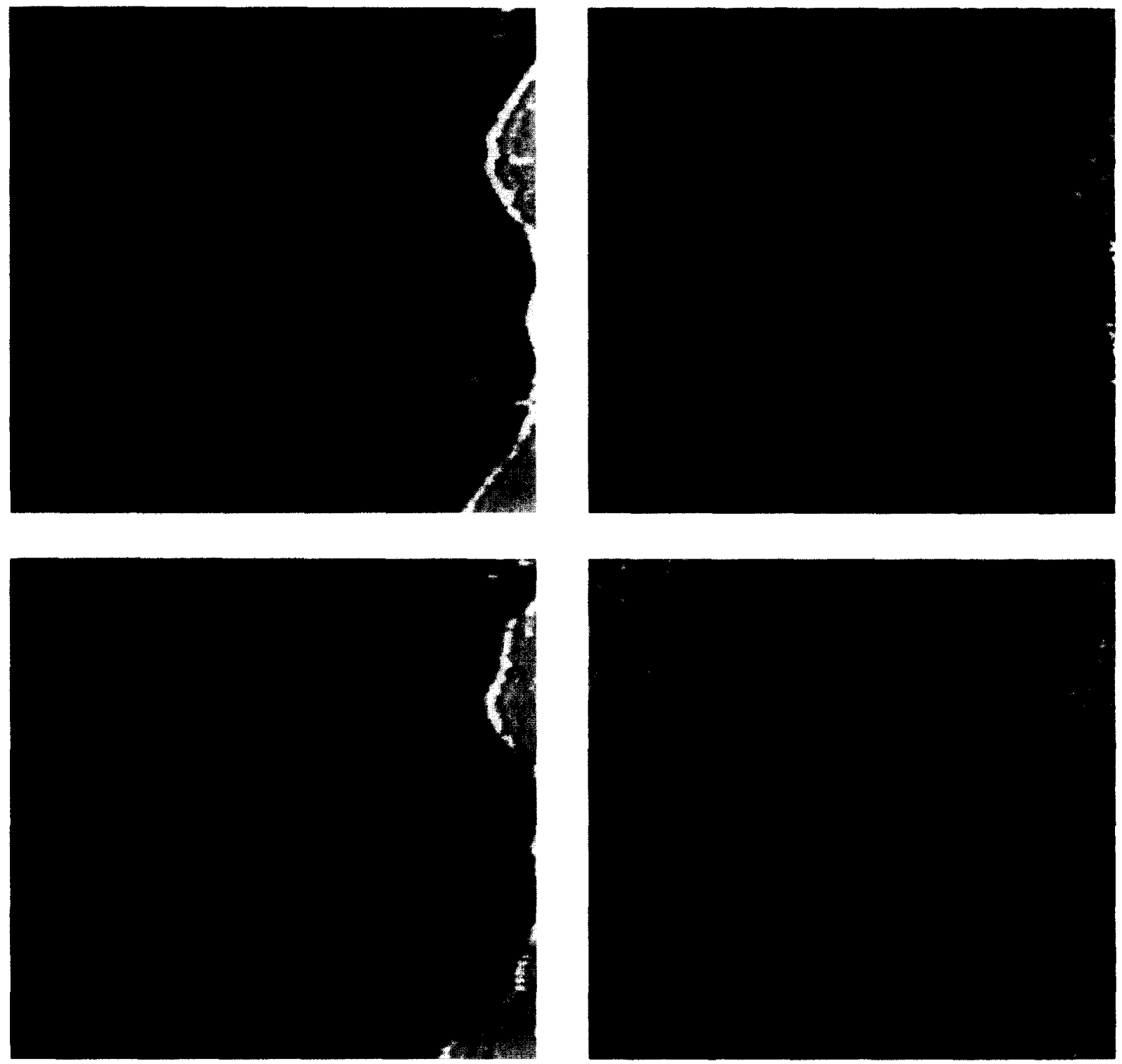

Fig. 6. In situ STM images of $\mathrm{n}-\mathrm{Si}(111)$ in $\mathrm{NaOH}$ showing the effect of a pulse of potential (see text for the procedure). The difference between the two frames reflects the effect of polarizing the surface for $5 \mathrm{~s}$ at rest bias. Left and right images correspond to the same portion of the surface but a different gray scale. See text for identification of sites A-E. Frames are $1400 \times 1400 \AA^{2}$.

cathodically so as to nearly stop the etching, like in Fig. 5. The tip was then immobilized immediately and the sample potential pulsed for $5 \mathrm{~s}$ to the rest bias while the regulation loop of the STM was simultaneously opened to avoid tip crashing. Immediately afterwards the second frame was recorded (at cathodic bias). Within this special "cook and freeze" procedure the same area is kept under observation and the differences between first and second images reflect the effect of the pulse. The advantage of the method is an accurate determination of the nucleation frequency of etch pits, since further pitting is stopped at cathodic polarization.

In Fig. 6, the two frames have been represented with two gray scales so as to resolve different 
aspects of the etching process. With a gray scale proportional to heights, the left-hand side column shows that some existing pits have grown (e.g. site A) and that new ones have been nucleated (e.g. site B). The dissolution occurs, however, mostly at step edges. The etch rate is $\sim 5 \AA \mathrm{min}^{-1}$ during the $5 \mathrm{~s}$ of the pulse. By applying the full gray scale to each of the atomic planes, the right-hand side column of Fig. 6 shows that the pulse generates isolated vertical $\equiv \mathrm{Si}-\mathrm{OH}$ on terraces. These foreign groups are the shallow depressions (depth $1 \AA$ and diameter $\sim 30 \AA$ ) observed on terraces and this STM contour arises from charge density effects, as discussed elsewhere [21]. Different types of $\equiv \mathrm{Si}-\mathrm{OH}$ are identified: (i) those which preexist to the pulse (e.g. site C), (ii) those which pre-exist and give rise to a pit (e.g. site D), and (iii) those which are generated by the pulse (e.g. site E). We stress that the depressions seen in Fig. 6 are not atomic vacancies.

Table 1 summarizes some data derived from Figs. 5 and $6 . N_{\mathrm{o}}$ is the density of $\equiv \mathrm{Si}-\mathrm{OH}$ and $F_{\mathrm{o}}$ is the nucleation frequency of isolated $\equiv \mathrm{Si}-\mathrm{OH}$. At the rest bias the value of $N_{\mathrm{o}}$ corresponds to the density after $5 \mathrm{~s}$. At cathodic bias, $N_{\mathrm{o}}$ was measured after several minutes and was nearly constant over long periods. $F_{\mathrm{p}}$ is the nucleation frequency of newly formed pits. Experimentally $F_{\mathrm{o}} \sim 30 F_{\mathrm{p}}$. The etch rates have been derived from the STM sequences. At cathodic bias two values are given. They correspond to Fig. 5 and to curve (b) of Fig. 13 (see below).

\subsection{Monte Carlo simulations}

The growth of a single triangle is shown in Fig. 7. An atom has been initially removed to

Table 1

Parameters derived from STM images

\begin{tabular}{lllll}
\hline Conditions & $\begin{array}{l}N_{\mathrm{o}} \\
\left(\mathrm{cm}^{-2}\right)\end{array}$ & $\begin{array}{l}F_{\mathrm{o}} \\
\left(\mathrm{cm}^{-2} \mathrm{~s}^{-1}\right)\end{array}$ & $\begin{array}{l}F_{\mathrm{p}} \\
\left(\mathrm{cm}^{-2} \mathrm{~s}^{-1}\right)^{\mathrm{b}}\end{array}$ & $\begin{array}{l}\text { Etch rate } \\
\left(\AA \mathrm{A} \mathrm{min}^{-1}\right)^{\mathrm{b}}\end{array}$ \\
\hline Rest bias & $\sim 2 \times 10^{11}$ & $\sim 1 \times 10^{11}$ & $3 \times 10^{9}$ & $5^{\mathrm{b}}$ \\
$\begin{array}{l}\text { Cathodic } \\
\text { bias }\end{array}$ & $\sim 1 \times 10^{11}$ & $\sim 1.5 \times 10^{8}$ & $-{ }^{\mathrm{a}}$ & $0.12^{\mathrm{b}}$ \\
& & & & $0.67^{\mathrm{c}}$
\end{tabular}

a Not measurable.

${ }^{b}$ Derived from STM observations (Figs. 5 and 6).

- Value corresponding to curve (b) of Fig. 13.
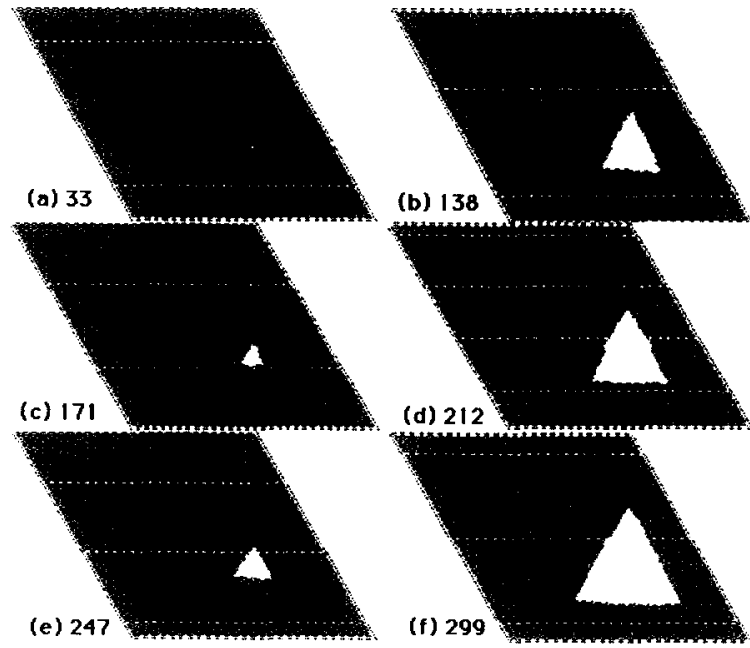

Fig. 7. Simulation showing the growth of a triangle. Parameters are: $k_{\mathrm{SM}}=0.01 \mathrm{~s}^{-1}$ and $k_{\mathrm{SD}}=1 \mathrm{~s}^{-1}$ and $k_{\mathrm{TM}}=0$. Time $(t / \mathrm{s})$ is as indicated in the figure.

initiate the pit and grow the largest possible pit. Parameters were $k_{\mathrm{SM}}=0.01 \mathrm{~s}^{-1}, k_{\mathrm{SD}}=1 \mathrm{~s}^{-1}$ and $k_{\mathrm{TM}}=0$ (no pitting allowed on the remaining surface). The time variation of the edge $L$ of the triangle is presented in Fig. 8. Triangular etch pits grow nearly exponentially in the initial stages (Fig. 8(a)) before a linear regime is reached (Fig. 8(b)), which fits Eqs. (1) and (2). It will be shown below that experimental data also follow the same behavior (see Fig. 13).

In Fig. 9, a complete row of atoms, parallel to the $[1 \overline{10}]$ direction, has been removed before the run to expose SM- and SD-terminated steps and investigate the attack on their edges. The sequence shows that the monohydride-terminated step remains nearly straight while the dihydride-terminated step is instantaneously roughened. Both migrate at a constant speed and Fig. 10 plots the speed as a function of the nucleation rate $k_{\mathrm{SM}}$. As long as $k_{\mathrm{SM}}<k_{\mathrm{SD}}$ the migration of the SM-terminated step is controlled by the nucleation of kinks. The motion of an SD step is independent of $k_{\mathrm{SM}}$ and only depends on $k_{\mathrm{SD}}$.

Fig. 11 simulates the effect of a pulse of potential. Parameters were $k_{\mathrm{TM}}=2 \times 10^{-3}$, $k_{\mathrm{SM}}=1 \times 10^{-2}$ and $k_{\mathrm{SD}}=1 \mathrm{~s}^{-1}$ during the first $3.4 \mathrm{~s}$. The run was then stopped after image (a) to enter a new set of parameters so as to account for the 

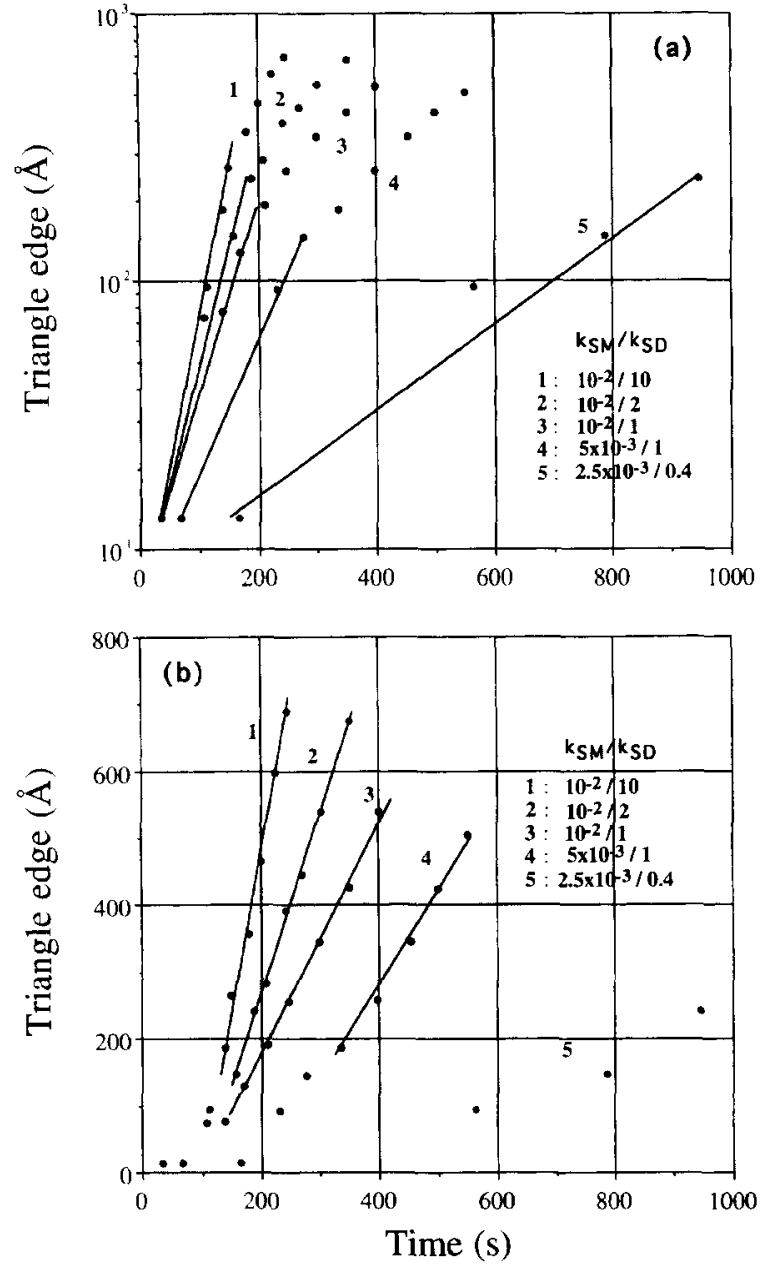

Fig. 8. Time dependence of the edge $L$ of triangular etch pits as measured from different simulations, like in Fig. 7. Values of $k_{\mathrm{SM}} / \mathrm{s}^{-1}$ and $k_{\mathrm{SD}} / \mathrm{s}^{-1}$ are as indicated in the figure. $k_{\mathrm{TM}}=0 \mathrm{~s}^{-1}$ in all cases.

cathodic polarization regime $\left(k_{\mathrm{TM}}=1 \times 10^{-5}\right.$, $k_{\mathrm{SM}}=1 \times 10^{-2}$ and $k_{\mathrm{SD}}=1 \mathrm{~s}^{-1}$ in images (b)-(d)). Like in Fig. 6, this technique enables one to measure accurately the density of pits as a function of $k_{\mathrm{TM}}$, since the density of pits remains nearly constant afterwards (see images (b)-(d)). In the $\log -\log$ scale, Fig. 12 indicates that the nucleation frequency of etch pits $F_{\mathrm{p}}$ and isolated TM vacancies $F_{\mathrm{o}}$ are both linearly dependent on $k_{\mathrm{TM}}$. The comparison of the two curves indicates that nearly one out of 50 of the TM vacancies generated gives rise to an etch pit. Parameters $k_{\mathrm{SM}}$ and $k_{\mathrm{SD}}$ had

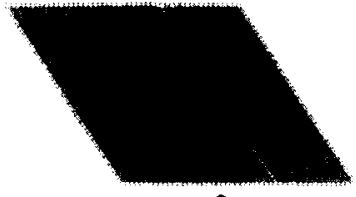

0

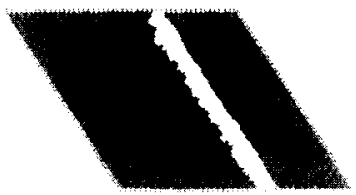

20

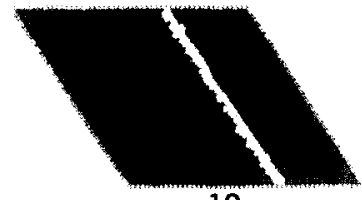

10

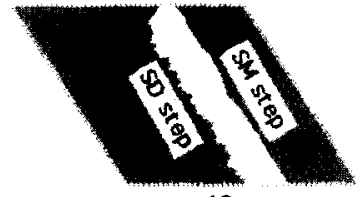

40
Fig. 9. Simulation showing the lateral migration of SM (righthand side) and SD (left-hand side) steps. Parameters are $k_{\mathrm{SM}}=0.01 \mathrm{~s}^{-1}$ and $k_{\mathrm{SD}}=1 \mathrm{~s}^{-1}$ and $k_{\mathrm{TM}}<=0$. Time $(t / \mathrm{s})$ is as indicated in the figure.

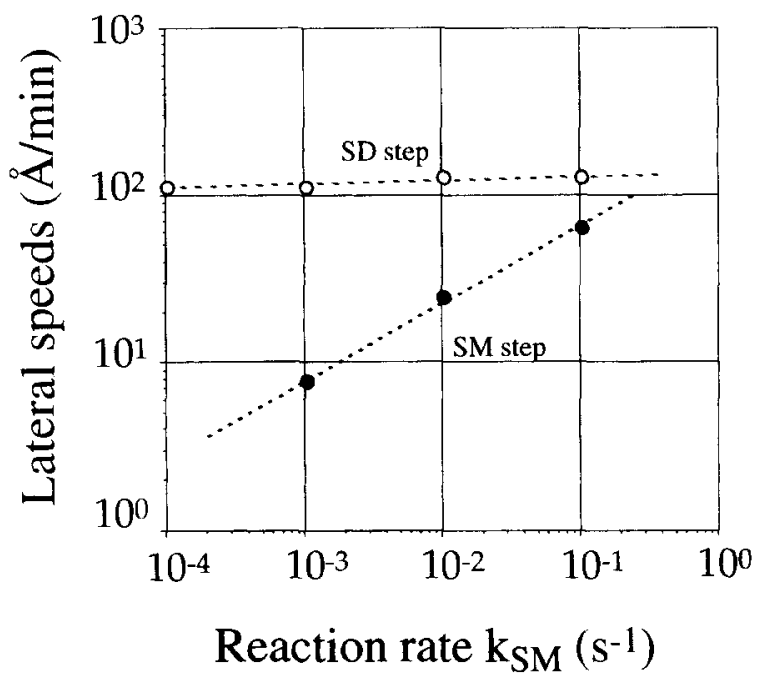

Fig. 10. Variations of $\mathrm{v}_{\text {step }}$ as a function of $k_{\mathrm{SM}}$ for $=\mathrm{Si}-\mathrm{H}_{2}$ (SD) and $\equiv \mathrm{Si}-\mathrm{H}(\mathrm{SM})$ steps.

little influence on the results. They mainly influenced the shape of etch pits and their growth rate.

\section{Discussion}

\subsection{Interpretation of the model}

Table 1 and Figs. 11 and 12 together yield detailed insights into the interpretation of the atomistic model. The chemical model (Fig. 1) indicates indeed that, on the atomic scale, Si 

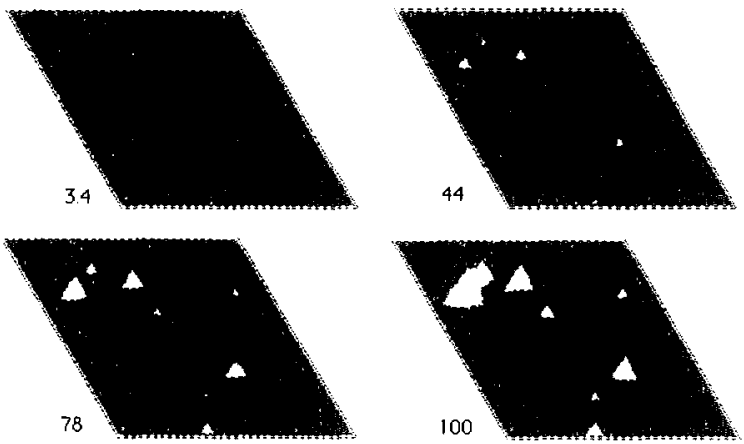

Fig. 11. Simulation of the effect of a pulse of potential. The pulse was featured with $k_{\mathrm{TM}}=2 \times 10^{-3} \mathrm{~s}^{-1}, \mathrm{k}_{\mathrm{SM}}=0.01 \mathrm{~s}^{-1}$ and $k_{\mathrm{SD}}=1 \mathrm{~s}^{-1}$ for the first $3.4 \mathrm{~s}$ (image (a)). The run was then stopped to input $k_{\mathrm{TM}}=1 \times 10^{-5} \mathrm{~s}^{-1}$ and let the pit grow (images (b)-(d)). Time $(t / \mathrm{s})$ is as indicated in the figure.

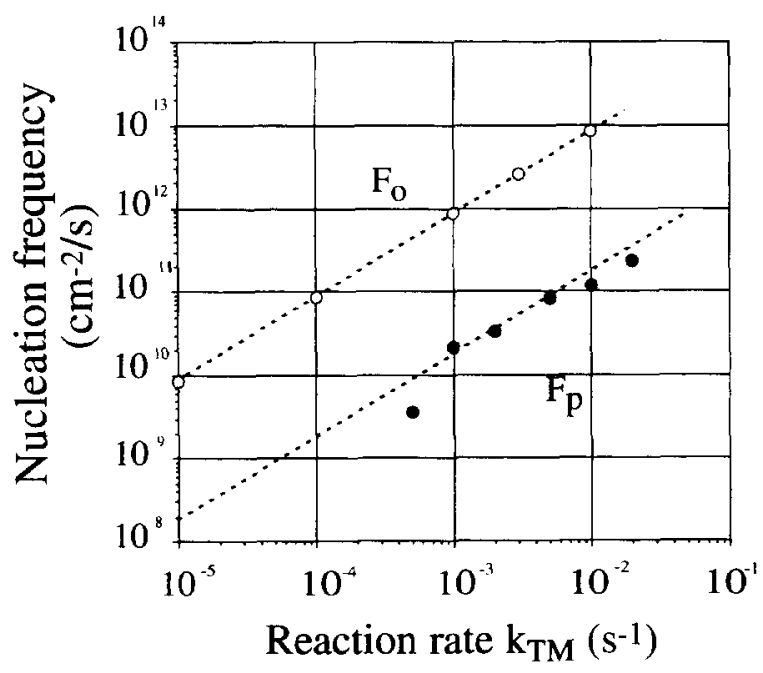

Fig. 12. Variations of the nucleation frequency of etch pits $F_{\mathrm{p}}$ and of single vacancies $F_{\mathrm{o}}$ as a function of the rate $k_{\mathrm{TM}}$. Other parameters are $k_{\mathrm{SM}}=0.01 \mathrm{~s}^{-1}$ and $k_{\mathrm{SD}}=1 \mathrm{~s}^{-1}$.

etching begins with the hydrolysis $\mathrm{Si}-\mathrm{H} \rightarrow \mathrm{Si}-\mathrm{OH}$ and that two routes are possible. The subsequent steps, which are not detailed here, are fast chemical steps, otherwise the surface could not remain H-terminated [18, 19,21]. For steric and orientational reasons, the hydrolysis step is slower on (111) terraces than at step edges. It nevertheless occurs occasionally, as Fig. 6 shows, and is certainly initiated by the electrochemical pathway (Fig. 1, bottom route) because the event occurs exclusively if this route is activated. At cathodic bias the electrochemical route is inhibited and almost no pitting occurs (see Fig. 5).

The removal of a terrace $\equiv \mathrm{Si}-\mathrm{OH}$ requires then that the three $\mathrm{Si}-\mathrm{Si}$ back-bonds be broken, which seems impossible when comparing the $\mathrm{Si}-\mathrm{Si}$ bond length $(2.35 \AA)$ and the hard-sphere diameter of water molecules $(2.45 \AA$ ). We therefore suggest that the isolated $T M$ vacancies of the simulation correspond to $\equiv \mathrm{Si}-\mathrm{OH}$ groups instead of missing atoms. This is supported by the fact that isolated TM vacancies are long-lived in Fig. 11 like terrace $\equiv \mathrm{Si}-\mathrm{OH}$ groups in Fig. 6 . In addition, the frequencies $F_{\mathrm{o}}$ and $F_{\mathrm{p}}$ are in a ratio of 50 (Fig. 12), which is fairly close to the experimental ratio of 30 (see Table 1). At step edge sites, such a detailed description is unnecessary since $\mathrm{Si}-\mathrm{H}$ bonds are more rapidly hydrolyzed and $\mathrm{Si}-\mathrm{OH}$ groups much shorter-lived.

\subsection{Validation of the model and determination of rate constants}

The time dependence of $L$ for triangles $\mathrm{A}-\mathrm{C}$ of Fig. 5 is plotted in Fig. 13. Curves depend critically on the origin of times. For triangles $\mathrm{B}$ and $\mathrm{C}$, the birth of triangles occurred in the top-left image in Fig. 5 (see ticks). For triangle A, whose birth was not resolved, we estimated it to be two or three images before the top-left image in Fig. 5. This assumption is confirmed by the fact that the data corresponding to this pit merge into those of pits $B$ and $C$ (a unique curve is indeed expected when accounting for the birth date of different pits). Curve (b) corresponds to a sequence recorded at less negative bias (hydrogen current $100 \mu \mathrm{A} \mathrm{cm} \mathrm{cm}^{-2}$ instead of $200 \mu \mathrm{A} \mathrm{cm}^{-2}$ for curve (a)) and for an etch rate of $0.67 \AA \mathrm{min}^{-1}$ ( Table 1). Within experimental uncertainty, Fig. 13 shows that triangles grow exponentially in the initial stages before a linear regime is reached, just like in the simulation (Fig. 8). That both results agree with Eqs. (1) and (2) validates the model and the algorithm used.

Observing the growth of bilayer-deep triangular etch pits appears, therefore, a relevant method for directly measuring $k_{\mathrm{SM}}$ and $k_{\mathrm{SD}}$. Combining Fig. 13 and Eqs. (1) and (2) yields indeed direct access to the constants $k_{\mathrm{SM}}$ and $k_{\mathrm{SD}}$. For short times $k_{\mathrm{SM}}$ is the slope of curve $\log L$ vs. $t$. For longer times the 

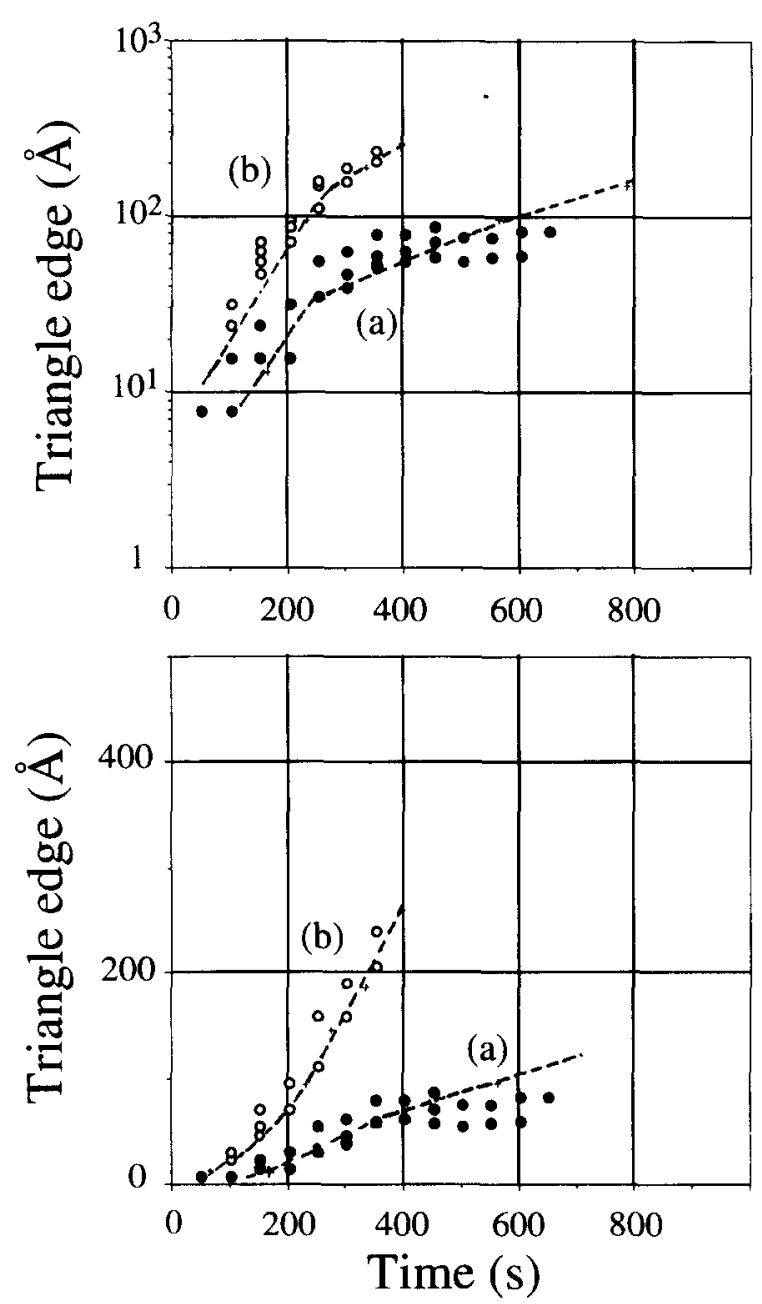

Fig. 13. Experimental time dependence of $L$ for triangles A-C in Fig. 5 (curve (a)). Curve (b) is derived from a sequence recorded with a smaller hydrogen current $\left(100 \mu \mathrm{A} \mathrm{cm}^{-2}\right)$. The origin of times is the birth of the nuclei (see text). Dotted lines are derived from simulation as in Fig. 8 with parameters: $k_{\mathrm{SM}}=5 \times 10^{-3} \mathrm{~s}^{-1}$ and $k_{\mathrm{SD}}=1 \mathrm{~s}^{-1} \quad$ (curve (a)) and $k_{\mathrm{SM}}=1 \times 10^{-3} \mathrm{~s}^{-1}$ and $k_{\mathrm{SD}}=0.5 \mathrm{~s}^{-1}$ (curve (b)).

slope of curve $L$ vs. $t$ is $\sqrt{ } k_{\mathrm{SM}} V k_{\mathrm{SD}}$. Numerically, $k_{\mathrm{SM}}=1 \times 10^{-3} \mathrm{~s}^{-1}$ and $k_{\mathrm{SD}}=0.1 \mathrm{~s}^{-1}$ for curve (a). These values are respectively $6.5 \times 10^{-3} \mathrm{~s}^{-1}$ and $0.8 \mathrm{~s}^{-1}$ for curve (b). Notice that both sets of values also agree with those derived from simulation (dotted lines). The comparison of curves (a) and (b) indicates further that $k_{\mathrm{SM}}$ and $k_{\mathrm{SD}}$ increase nearly linearly with the etch rate (see Table 1 for values of etch rates).
The determination of $k_{\mathrm{TM}}$ can also be made directly from experiments. The technique used in Fig. 6 can be repeated for different pulse heights, as in Ref. [4], to plot the bias dependence of $F_{\mathrm{p}}$. Fig. 14 shows that $F_{\mathrm{p}}$ is an exponential law of the bias. Combining Figs. 12 and 14 , where $\log \left(F_{\mathrm{p}}\right)$ is respectively proportional to $U$ and to $\log \left(k_{\mathrm{TM}}\right)$, yields $\quad k_{\mathrm{TM}}=k_{\mathrm{TM}}^{\circ} \exp \left[a\left(U-U_{\mathrm{o}}\right) / k T\right], \quad$ with $U_{\mathrm{o}}=-1.1 \mathrm{~V}$ being the open circuit potential, $a=0.21$ the transfer coefficient and $k_{\mathrm{TM}} \sim 1 \times 10^{-3} \mathrm{~s}^{-1}$.

The exponential dependence of $k_{\mathrm{TM}}$ is a further validation of our model, since this agrees perfectly with the view that, on a (111) terrace, etch pits must be initiated by the electrochemical formation of a vertical $\mathrm{Si}-\mathrm{OH}$ group as discussed above (see Fig. 1 and Ref. [4] for more details).

Table 2 (left-most column) summarizes the rate constants for etching at rest bias in $\mathrm{NaOH}$. The value of $k_{\mathrm{TM}}$ is derived directly from the experiment in Fig. 6. Values of $k_{\mathrm{SD}}$ and $k_{\mathrm{SM}}$ are, by contrast, extrapolations from the measurements made at cathodic bias (Fig. 5). Experimentally, it is indeed impossible to follow the growth of etch pits at the rest bias. Assuming that the relationship between the $k_{i}$ values $(i=\mathrm{SD}, \mathrm{SM})$ and the etch rate (see Table 1$)$ is still nearly proportional when approaching the rest bias leads to $k_{\mathrm{SM}}$ and $k_{\mathrm{SD}} \sim 0.04 \mathrm{~s}^{-1}$ and $\sim 5 \mathrm{~s}^{-1}$. We are aware that this is only an estimate and not a precise determina-

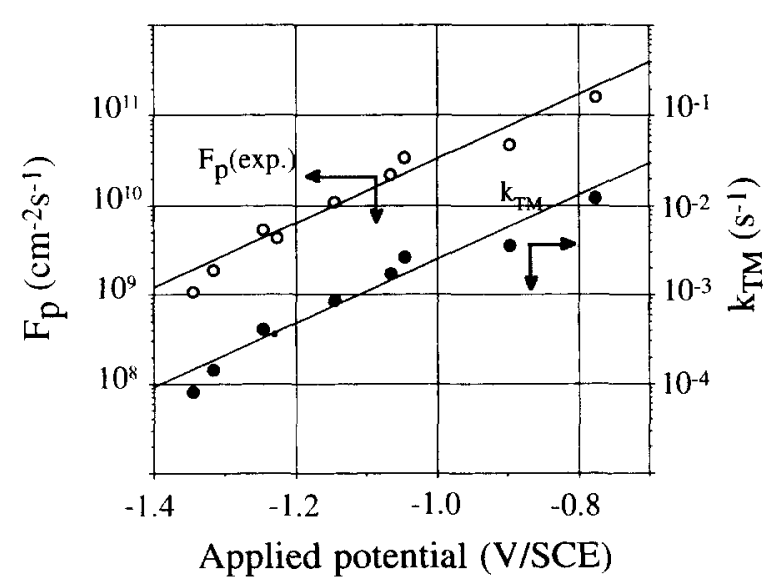

Fig. 14. Bias dependence of $F_{\mathrm{p}}(U)$ on $\mathrm{n}-\mathrm{Si}(111)$ in $\mathrm{NaOH}$ (after Ref. [19]). The bias variation of $k_{\mathrm{TM}}$ is derived from the combination of $F_{\mathrm{p}}(U)$ with $F_{\mathrm{p}}\left(k_{\mathrm{TM}}\right)$ (see Fig. 12). 
Table 2

Parameters derived from the combined STM and simulation approach. Activation energies for removing the different sites are compared with those measured on corresponding faces

\begin{tabular}{|c|c|c|c|c|}
\hline Site $(i)$ & $\begin{array}{l}\text { Rate constant } k_{i}{ }^{a} \\
\left(\mathrm{~s}^{-1}\right)\end{array}$ & Bias potential dependence & $\begin{array}{l}E_{\mathrm{a}}(\mathrm{eV}) \\
\text { (simulation) }\end{array}$ & $\begin{array}{l}E_{\mathrm{a}}(\mathrm{eV}) / \text { orientation } \\
(\text { experimental })^{\mathrm{c}}\end{array}$ \\
\hline $\mathrm{TM}$ & $1 \times 10^{-3}$ & Exponential & $\begin{array}{l}0.89^{\mathrm{d}} \\
0.97^{\mathrm{c}}\end{array}$ & $0.78 /(111)$ \\
\hline SM & 0.04 & $-{ }^{b}$ & 0.79 & $0.68 /(110)$ \\
\hline SD & 5 & $-^{\mathbf{b}}$ & 0.67 & $0.65 /(100)$ \\
\hline
\end{tabular}

${ }^{a}$ Under chemical etching conditions (i.e. at rest bias).

b Nearly linear (see text).

c Activation energy to generate a triangular pit

d Activation energy to generate an $\equiv \mathrm{Si}-\mathrm{OH}$.

e Values taken from Ref. [8] for $24 \% \mathrm{NaOH}$.

tion. Table 2 calls for a remark. The ratios $k_{\mathrm{TM}} / k_{\mathrm{SM}} \sim 2.5 \times 10^{-2}$ and $k_{\mathrm{TM}} / k_{\mathrm{SD}} \sim 2 \times 10^{-4}$ are very similar to those found by Higashi and Chabal in $40 \% \quad \mathrm{NH}_{4} \mathrm{~F}$ [1] (values are respectively $(1-2) \times 10^{-2}$ and $\left.(2-10) \times 10^{-4}\right)$ which suggests that the rate constants are about ten times smaller in this solution than in $\mathrm{NaOH}$ because the macroscopic etching $R$ is ten times smaller $\left(R \sim 20 \AA \min ^{-1}\right.$ and $\sim 2 \AA \min ^{-1}$ respectively in $\mathrm{NaOH}$ and $\left.40 \% \mathrm{NH}_{4} \mathrm{~F}[4]\right)$.

\subsection{Activation energy' of the reaction: comparison with macroscopic data}

Writing reaction rates as $k_{i}=k_{i}^{\circ}$ $\exp \left(a_{i}\left(U-U_{\mathrm{o}}\right) / k T\right)$ with $i=\mathrm{TM}, \mathrm{SM}$ and SD, the activation energies $E_{\mathrm{a}}$ can be calculated using the expression of $k_{i}^{\circ}=v \exp \left(-E_{\mathrm{a}} / k T\right)$, with $v=10^{12} \mathrm{~s}^{-1}$ the vibrational frequency of surface atoms. The value of $a_{\mathrm{TM}}$ is given above. The transfer coefficients $a_{\mathrm{SM}}$ and $a_{\mathrm{SD}} \sim 0$, since the corresponding reaction rates vary only slowly with the bias potential (see Table 2 and related discussion).

Table 2, collects the values of $E_{\mathrm{a}}$. It is interesting to remark that they are close to those found by Camon et al. [7] to break $\mathrm{Si}-\mathrm{Si}$ bonds of similar atomic configurations. Our data also give the activation energy to create a terrace $\equiv \mathrm{Si}-\mathrm{OH}$ group $(0.89 \mathrm{eV})$ and indicate that the energy barrier is $0.06 \mathrm{eV}$ larger $(0.97 \mathrm{eV})$ since only $3 \%$ of $\equiv \mathrm{Si}-\mathrm{OH}$ lead to a pit.

Since TM, SM and SD sites respectively have the coordination of atoms on ideal (111), (110) and (100) planes, Table 2 tentatively compares $E_{\mathrm{a}}$ with macroscopic determinations performed on these three low-index planes. The excellent agreement between the $0.67 \mathrm{eV}$ found for removing an SD atom and the experimental $0.65 \mathrm{eV}$ found for the (100) [8] justifies the linear extrapolation made to estimate the constant at rest bias and suggests that this event is mainly chemical. There is a $0.1 \mathrm{eV}$ discrepancy for SM sites. Obviously we underestimate $k_{\mathrm{SM}}$ at rest bias. We attribute this to the fact that the removal of SM sites probably partly follows the electrochemical route in Fig. 1 (as for TM sites) and that $k_{\mathrm{SM}}$, therefore, varies more rapidly than anticipated with the bias. The activation energy to bind an $\mathrm{OH}$ group may be compared to $a b$ initio calculations to bind an $F$

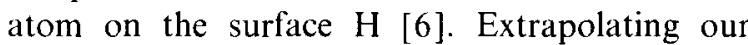
$0.89 \mathrm{eV}$ to the case of $\mathrm{NH}_{4} \mathrm{~F}$, where the etch rate is ten times slower, yields $0.95 \mathrm{eV}$ (see Section 5.2) against $1.2 \mathrm{eV}$ found in Ref. [6]. Last, the discrepancy between the $0.97 \mathrm{eV}$ barrier to nucleate a pit with the value of $E_{\mathrm{a}}$ measured on the (111) face is not a big surprise. In fact the macroscopic determination involves the effects of many steps and defects, which is not so in our case.

\subsection{Critical nucleus: comparison with the classical nucleation picture}

As discussed above in Section 5.1, isolated TM vacancies are vertical $\equiv \mathrm{Si}-\mathrm{OH}$ groups. We therefore speculate that the following mechanism creates an etch pit. After several $\equiv \mathrm{Si}-\mathrm{OH}$ have been 
generated next to each other, they begin to condense and form $\mathrm{Si}-\mathrm{O}-\mathrm{Si}$ bridges. This promotes lattice strains which favor the attack of the surface by water and enables the insertion of molecules to break an $\mathrm{Si}-\mathrm{Si}$ back-bond. In other words, etch pits originate from the formation of a small cluster of suboxide which is removed chemically as one entity, leaving a monolayer-deep pit of only three atoms. Simulation indeed confirms that only such triple vacancies may grow as a triangular etch pit. In the classical two-dimensional nucleation picture used in crystal growth, the critical nucleus would therefore be three atoms large.

It is interesting to compare this estimate with the classical theory of two-dimensional nucleation of crystal growth. Transposition to the case of dissolution, where cavities are generated instead of islands, the barrier to create a pit arises from two competing energy contributions. The first one stems from the fact that the solid material is dissolved (in the case of silicon, etching after chemical reaction). This energy term is equal to the number of atoms removed times the thermodynamic driving force $\Delta \mu$. It is also proportional to the area of the pit. The second term arises from the fact that the generation of a pit creates steps on the surface. This costs an energy equal to the length of the step times the step free energy $i$. The second term is proportional to the diameter of the pit. The free energy of a pit as a function of the pit radius then has a maximum at a pit radius $R^{*}$ with a height $\Delta G^{*}$. We have $R^{*}=\gamma / \Delta \mu$ and $\Delta G^{*}=\gamma^{2} / \Delta \mu$, with $R^{*}$ the radius of the critical nucleus [22]. The idea of the energy barrier to create a critical nucleus involves, therefore, that nuclei of radius smaller than $R^{*}$ most probably disappear, because this is the most convenient way to minimize the free energy of the system. Conversely, once a pit is larger than $R^{*}$ it grows freely.

There are two basic assumptions for the classical nucleation picture that must hold in order to be applicable to silicon etching. First, the $\langle 111\rangle$ surface must be flat or, in other words, the surface must be at a temperature below the roughening temperature. This assumption is necessary because a rough surface has a step free energy $\gamma=0$. Such faces have no energy barriers. This condition is met since we believe from our STM images that there is no doubt that the $\langle 111\rangle \mathrm{Si}$ face is flat during anisotropic chemical etching. The second assumption concerns the reversibility of the process. This is necessary, because thermal fluctuations must allow the system to choose freely how to minimize its energy once a pit of radius $R<R^{*}$ has formed.

If we use numerical values of the thermodynamic driving force ( $\Delta \mu=3 \mathrm{eV}$, as estimated roughly from the heat of oxidation of silicon) and for the nucleation barrier $\left(\Delta G^{*}=1 \mathrm{eV}\right.$, as estimated from simulations, see above), we find $R^{*}=1.7$ atomic units and $\hat{\imath}=1.7 \mathrm{eV}$. This result agrees with observations, because simulation indicates that the critical nucleus corresponds to about three TM atomic vacancies. There are, however, some problems. If $\Delta \mu$ is actually as large as $3 \mathrm{eV}(=100 k T$ ? $)$ the reaction is absolutely irreversible. This last point is actually well verified, since redeposition of $\mathrm{Si}$ is impossible. $\Delta \mu$ may, however, be greatly overestimated. In the chemical reaction silicon is indeed oxidized, but several water molecules are also dissociated and each dissociation step costs an energy of $2.4 \mathrm{eV}$. This thereby reduces $\Delta \mu$ and will change the estimate of $R^{*}$.

\section{Conclusion}

In summary, an atomistic model has been presented for $\mathrm{Si}$ anisotropic etching. Validation of the model was assessed from the comparison of computer simulations with real-time STM imaging. On the atomic scale, the reaction of dissolution seems to be uniquely affected by the coordination of atoms while the neighboring surface atoms have no or little influence. The difficulty of nucleating triangular etch pits on terraces has also been discussed in terms of formation of a small cluster of suboxide prior to the removal of atoms. Comparison with theory of nucleation confirmed this estimate. This study also proposed a simple method to measure the different reaction rates and activation energies on the atomic scale. The activation energy of the $\mathrm{Si}-\mathrm{H} \rightarrow \mathrm{Si}-\mathrm{OH}$ step could be measured on terrace monohydrides, and results suggest that this step is at the root of $\mathrm{Si}$ anisotropic 
etching. Applications of results will be the prediction of surface microstructures of different crystal planes and examining the rôle of different parameters when generating nanostructures by selective etching of patterns of nanometer dimensions.

\section{Appendix A: Algorithm of simulation}

The model removes atoms from a (111) crystal monolayer, defined as hexagonal sites (see text and Fig. 2). The algorithm is based on the Monte Carlo method [23]. A two-stage process is considered. The first one determines randomly the family (TM, SM or SD, see Fig. 2) from which the atom will be removed. The second one removes randomly one atom from the pre-determined family. Computation involves three parameters $k_{i} / \mathrm{s}^{-1}(i=$ TM, SM or SD) which are the rates of the dissolution of sites of type $i$.

At time $t$, there are $n_{i}(t)$ sites of type $i$ whose positions are tabulated. The instantaneous total dissolution rate is $\sum_{i} k_{i} n_{i}(t)$ (with $i=\mathrm{TM}, \mathrm{SM}, \mathrm{SD}$ ). One site is therefore removed, on the average, during the time interval $\Delta t=1 / \sum_{i} k_{i} n_{i}(t)$. This time interval is used to increment time in computation. To avoid unsuccessful attempts and to save computing time, the algorithm considers that exactly one atom is removed during $\Delta t$ (it was verified that this did not affect the results). It is important to note that the $\Delta t$ is not a constant throughout simulation, since the populations $n_{i}$ are varying with corrosion time. The removed atom will be of type $j(j=\mathrm{TM}, \mathrm{SM}$ or $\mathrm{SD})$ if the random number $x(0<x<1)$ is

$\sum_{i<j} v_{i} \leq x<\sum_{i \leq j} v_{i}$

with

$v_{i}=\frac{k_{i} n_{i}(t)}{\sum_{j=\mathrm{TM} . \mathrm{SM} . \mathrm{SD}} k_{j} n_{j}(t)}$.

The position of the removed site is then simply chosen at random among the populations $n_{j}(t)$, since all sites $j$ are equiprobable.

\section{References}

[1] G.S. Higashi, Y.J. Chabal, in: W. Kern (Ed.), Handbook of Semiconductor Wafer Cleaning Technology, Noyes Publications, Park Ridge, 1993.

[2] P. Jakob, Y.J. Chabal, J. Chem. Phys. 95 (1991) 2897.

[3] P. Allongue, V. Kieling, H. Gerischer, Electrochim. Acta 40 (1995) 1353.

[4] P. Allongue, V. Costa-Kieling, H. Gerischer, J. Electrochem. Soc. 140 (1993) 1019.

[5] J.R. Roche, M. Ramonda, F. Thibaudau, Ph. Dumas, Ph. Mathiez, F. Salvan, P. Allongue, Microsc. Microanal. Microstruct. 5 (1994) 291.

[6] G.W. Trucks, K. Raghavachari, G.S. Higashi, Y.J. Chabal, Phys. Rev. Lett. 65 (1990) 249.

[7] H. Camon, Z. Moktadir, M. Djafari-Rouhani, E-MRS Spring Meet. Proc., 1995, G-V-5.

[8] H. Seidel, L. Csepregi, A. Heuberger, H. Baumgärtel, J. Electrochem. Soc. 137 (1990) 3612.

[9] D.L. Kendall, Annu. Rev. Mater. Sci. 9 (1979) 373.

[10] P. Allongue, V. Kieling, H. Gerischer, J. Phys. Chem. 99 (1995) 9472 .

[11] G.J. Pietsch, U. Köhler, M. Henzler, J. Appl. Phys. 73 (1993) 4797

[12] H.E. Hessel, A. Feltz, U. Memmert, R.J. Behm, Chem. Phys. Lett. 186 (1991) 275.

[13] P. Jakob, Y.J. Chabal, K. Raghavachari, R.S. Becker, A.J. Becker, Surf. Sci. 275 (1992) 407.

[14] Y. Morita. H. Tokumoto, Appl. Phys. Lett. 67 ( 1995) 2654

[15] P. Allongue, J. Kasparian. Microsc. Microanal. Microstruct. 5 (1994) 257.

[16] J. Kasparian, P. Allongue, in preparation.

[17] A. Venkateswara Rao, F. Ozanam, J.-N. Chazalviel, J. Electrochem. Soc. 138 (1991) 153.

[18] J. Rappich. H.J. Lewerenz, H. Gerischer, J. Electrochem. Soc. 141 (1994) L187.

[19] P. Allongue, V. Kieling, H. Gerischer, J. Electrochem. Soc. 140 (1993) 1008. P. Allongue, H. Brune, H. Gerischer. Surf. Sci. 275 ( 1992 ) 414.

[20] P. Allongue, Scanning Tunneling Microscopy of Semiconductor Electrodes, in: H. Gerischer, C.W. Tobias (Eds.), Advances in Electrochemical Science and Engineering, vol. 4, VCH, Weinheim, 1995, Chapter 1, pp. 1-66.

[21] P. Allongue, Phys. Rev. Lett. 77 (1996) 1986.

[22] M. Elwenspoek, J. Electrochem. Soc. 140 (1993) 2075.

[23] C.S. Kholi, M.B. Ives, J. Cryst. Growth 16 (1972) 123. 